\title{
A novel nonsense EIF1AX mutation identified in a thyroid nodule histologically diagnosed as oncocytic carcinoma
}

\author{
Marialuisa Sponziello ${ }^{1}$ - Gabriella Silvestri ${ }^{2}$ - Antonella Verrienti ${ }^{1}$ - Alessia Perna ${ }^{2}$ - Francesca Rosignolo ${ }^{1}$. \\ Chiara Brunelli ${ }^{3}$. Valeria Pecce ${ }^{1} \cdot$ Esther Diana Rossi $^{3} \cdot$ Celestino Pio Lombardi ${ }^{4} \cdot$ Cosimo Durante $^{1}$. \\ Sebastiano Filetti ${ }^{1} \cdot$ Guido Fadda $^{3}$
}

Received: 24 January 2018 / Accepted: 17 April 2018

(c) Springer Science+Business Media, LLC, part of Springer Nature 2018

\section{Introduction}

Thyroid nodules are common in the general population (prevalence 16-68\% depending on the screening method and population analyzed) [1]. Over $90 \%$ of the nodules detected are benign and will never undergo transformation $[2,3]$. Accurate preoperative identification of these lesions reduces the risk of unneeded surgery [2]. Preoperative diagnoses of malignancy are based mainly on suspicious ultrasonographic findings verified by cytological examination of fine-needle aspirates (FNA) [4, 5]. For cytologically indeterminate nodules $(25 \%)[4,6]$, molecular analyses of the aspirates can often help to identify or exclude malignancy [7]. A promising tool for this purpose is the ThyroSeq v2 mutation panel [8], which can identify singlenucleotide variants, indels, and gene fusions currently known to drive thyroid carcinogenesis, including several recently identified by The Cancer Genome Analysis (TCGA) network [9]. Using a similar NGS-based approach, we identified a novel EIFIAX mutation in a cytologically indeterminate thyroid nodule that ultimately proved to be an angioinvasive oncocytic thyroid carcinoma.

Antonella Verrienti

antonella.verrienti@uniroma1.it

1 Department of Internal Medicine and Medical Specialties, Sapienza University of Rome, Rome, Italy

2 Institute of Neurology, Foundation "Agostino Gemelli” University Hospital, Rome, Italy

3 Division of Anatomic Pathology and Histology, Foundation "Agostino Gemelli" University Hospital, Rome, Italy

4 Division of Endocrine Surgery, Foundation "Agostino Gemelli" University Hospital, Rome, Italy

\section{Methods}

\section{Case presentation}

A 55-year-old Italian man underwent thyroidectomy for a $2.8-\mathrm{cm}$ nodule in the right lobe that was cytologically indeterminate (TIR 3B-oncocytic nodule in the Italian Reporting System, corresponding to Bethesda class IVHürthle cell nodule) (Fig. 1a). The nodule was solid, wellcircumscribed, and thickly encapsulated. Histological examination revealed a predominance of oncocytic cells in a microfollicular, trabecular pattern (Fig. 1b). Extensive sampling identified multiple foci of capsular and vascular invasion (Fig. 1c, d, respectively). The final diagnosis was angioinvasive oncocytic thyroid carcinoma (pT2 Nx $\mathrm{Mx})$. It was administered a therapeutic activity of radioactive iodine for adjuvant purpose and post-therapeutic whole-body scan was negative. Sixteen months of followup have not revealed any evidence of residual/recurrent disease.

The patient had been diagnosed with myotonic dystrophy type I (DM1, MIM \#160900) [10], an autosomal dominant neuromuscular disorder that also affected his mother, brother, two sisters, and two nephews. None of the patient's first-degree relatives, regardless of their DM1 status, had histories of thyroid cancer or other malignancies. Molecular testing for DM1 revealed an abnormal CTG expansion in the 3' UTR of DMPK (130-350 CTG repeats; negative values: 5-35) [11].

\section{Molecular analysis of the thyroid nodule}

FNA of the thyroid nodule was processed for cytology with ThinPrep5000 ${ }^{\mathrm{TM}}$ system (Hologic Co.). The material remaining after cytological analysis was used for molecular profiling. Genetic analysis was performed on the Ion S5 system (Thermo Fisher Scientific) using two custom 
$\mathbf{a}$

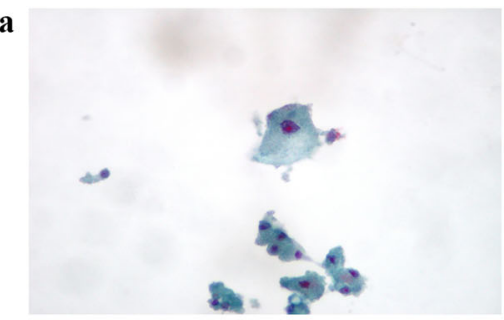

b

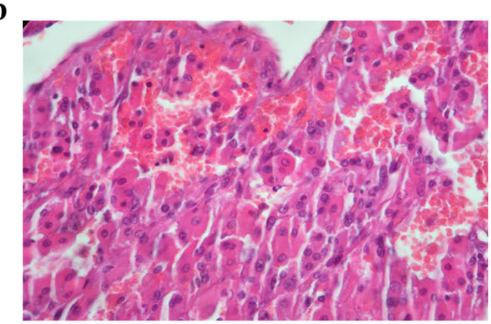

c

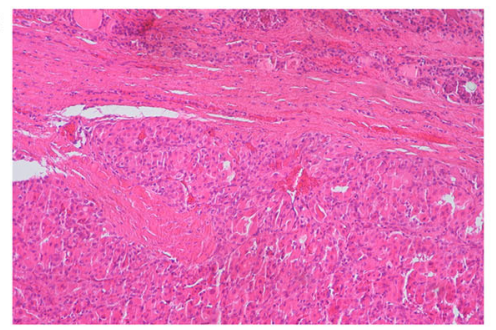

d

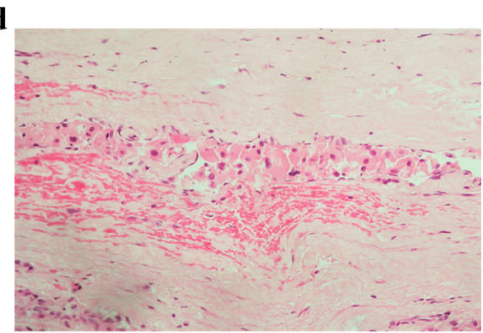

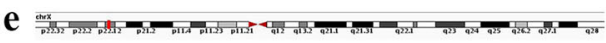

p.Gln130*

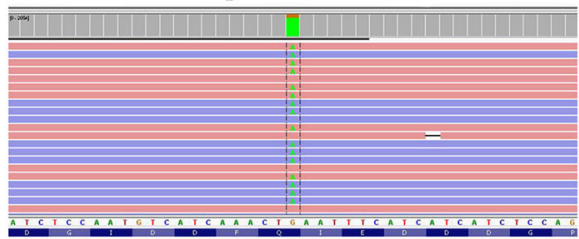

f

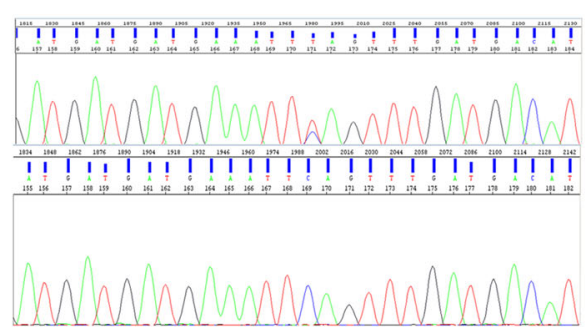

Fig. 1 EIFIAX mutation in an oncocytic thyroid carcinoma. a Nodule cytologically classified as indeterminate TIR 3B (oncocytic nodule) of the Italian Reporting System corresponding to Bethesda class IVHürthle cell nodule (Papanicolau-stained slide, $\times 500$ ) b, c, d Definitive histological diagnosis of angioinvasive oncocytic thyroid carcinoma (H\&E-stained sections: (b) morphological details, $\times 250$; (c) foci of capsular invasion, $\times 125$; (d) foci of vascular invasion, $\times 125$ ). e IGV visualization of the EIF1AX variant (chrX:20148675G $>A$; p. Gln130*). Pink and blue bars represent forward and reverse read strands, respectively. f Sanger Sequencing validation of the EIF1AX variant on tumor (upper panel) and normal (lower panel) thyroid tissues from FFPE samples
NGS multi-gene panels, which tested for single-nucleotide variants/small indels (DNA panel) and gene fusions (RNA panel) involving well-known thyroid cancer-related genes (e.g., BRAF, RAS, EIFIAX, TERT, RET/PTC, and PAX8/ $P P A R G$ fusion) and others from our in-house database. DNAs and RNAs were isolated simultaneously using the All Prep DNA/RNA kit (QIAGEN) and measured with the Qubit dsDNA/RNA High Sensitivity assays (Thermo Fisher Scientific). Genes for the custom panel were selected based on literature data $[8,9,12]$ and our in-house database, and custom primers for their targeted amplification were designed with the Ion AmpliSeq ${ }^{\mathrm{TM}}$ Designer (Thermo Fisher Scientific). Two libraries were created from $20 \mathrm{ng}$ of DNA and 20 ng of RNA. The targeted amplification products were partially digested, phosphorylated, and ligated to Ion P1 adapter and Ion Xpress ${ }^{\mathrm{TM}}$ barcodes (Thermo Fisher Scientific). Libraries were pooled and clonally amplified on the Ion One Touch2 System. Sequencing was performed using Ion S5 sequencing solution on an Ion 530 chip (Thermo Fisher Scientific). Data were analyzed with Variant Caller v5.2, annotated with Ion Reporter 5.6 and wANNOVAR software and prioritized on the basis of their population frequency (Minor allele frequency <0.005). Variants were called when the position was covered by over 500 reads. The lower detection limits were set at 5\% for SNVs and $15 \%$ for small indels. Predicted variant deleteriousness was assessed with wANNOVAR (http://wannovar. wglab.org/).

NGS results and the germ line/somatic status of each variant were further validated by Sanger sequencing analysis (as described elsewhere [13]) of formalin-fixed, paraffin-embedded (FFPE) surgical specimens of normal and neoplastic thyroid tissues. Primer sequences are available upon request.

\section{Results}

Our amplicon-based NGS analysis of the cytologically indeterminate thyroid nodule FNA revealed a single somatic mutation involving exon 6 of the eukaryotic translation initiation factor 1A (EIF1AX) gene (NM_001412, c.C388T, p.Gln130*) (Fig. 1e), which is located on chromosome X. No mutations were detected in the well-known thyroid cancer-related genes, including BRAF, RAS, RET/PTC, $P A X 8 / P P A R G$, and TERT. The EIFIAX mutation was found in $82 \%$ of the reads. Sanger sequencing confirmed its presence in FFPE tumor slices but not in normal tissue from the unaffected lobe (Fig. 1f). The high allele frequency (82\%) can be attributed to EIFIAX's localization on the X chromosome. The presence of two alleles probably reflects contamination from non-tumor cells. The deleteriousness of the EIF1AX stopgain variant was predicted by MutationTaster, CADD, DANN, Fathmm-MKL, GenoCanyon, GERP++, phyloP100way_mammalian, pastCons20way_mammalian, and SiPhy_29way_logOdds (data not shown). Sequencing of DNA from the patient's normal thyroid tissue also revealed a germ line missense mutation in CHEK2 (NM_007194.3, c.1067C >T, p.Ser356Leu), which controls cell cycling and DNA repair. 


\section{Discussion}

EIF1AX mutations have been documented in papillary, poorly differentiated, and anaplastic thyroid carcinomaswith or without known driver mutations affecting $R A S$ or TP53-and, more recently, in benign thyroid nodules [14] and a Hürthle cell carcinoma [15]. Most of these mutations affected the sequence encoding the $\mathrm{N}$-terminal tail of eIF1A. $[9,12,14]$, whereas the novel mutation we found, $p$. Gln130*, involves the sequence encoding eIF1A's Cterminal. Few cancer-related mutations have been described in this area, and the only one reported in thyroid cancer is the A113_splice mutation [9, 12, 14], which seems to be the most prevalent EIFIAX mutation in thyroid malignancy [14]. Moreover, p.Gln130* is a nonsense mutation that results in a truncated protein. Nonsense EIFIAX mutations have been reported in cancers of the colon (p.Gly $8 *$ ), esophagus (p.Glu99*), breast (p.Glu117*), and vagina (p. Glu139*) (http://www.cbioportal.org/, accessed January 2018). However, the p.Gln130* variant is the first eIF1A nonsense mutation reported in thyroid cancer and the second EIFIAX mutation of any type in an oncocytic thyroid carcinoma. Importantly, because EIFIAX is located on the X chromosome, our male patient's tumor harbored only truncated eIF1A proteins. The potential impact of this alteration is unclear. However, studies in yeast indicate that the Cterminal is necessary for eIF1A's interaction with EIF5B, which is essential for its wild-type translation in vivo. Yeast cells harboring eIF1A lacking the final 24 residues display decreased rates of translation initiation in vivo [16].

The possible role of our patient's germ line missense mutation in CHEK2 is also unclear. Germ line mutations involving CHEK2 have been implicated in inherited susceptibility to cancers of the breast, ovary, and prostate [17]. The p.Ser356Leu variant itself is annotated in ClinVar (rs121908703) as having "uncertain clinical significance." However, none of the patient's first-degree relatives had histories of any type of cancer, including that of the thyroid. Moreover, the germ line CHEK2 p.Ser356Leu was also found in a brother of the patient's with DM1, which is reportedly associated with an increased risk of thyroid cancer [18-20]. However, it was also harbored by a second sibling without DM1 (data not shown), which also argues against the possibility of an association between CHEK2 and $D M P K$ gene.

In summary, we identified a novel EIFIAX mutation (c.388C $>\mathrm{T}$ ) in a nodule ultimately diagnosed as oncocytic thyroid carcinoma. Functional studies are needed to better define its role in thyroid tumorigenesis. The possibility that it is a new driver of thyroid carcinogenesis is suggested by the absence of other somatic driver mutations in the FNA specimen we analyzed. It is also consistent with the fact that the mutation results in a truncated eIF1A protein lacking the
C-terminal, which appears to play a key role in eukaryotic translation.

Acknowledgements The study was supported by the Umberto Di Mario Foundation, the "Sapienza" University of Rome (grant RM11715C7DD0EF56 to S.F.) and by PRIN 2015 (grant Prot. 2015HPMLFY to C.D.).

\section{Compliance with ethical standards}

Ethics approval All procedures involving humans were in accordance with the ethical standards of the institutional and/or national research committee and with the 1964 Helsinki declaration and its later amendments or comparable ethical standards.

Informed consent Informed consent was obtained from all subjects included in the study.

Conflict of interest The authors declare that they have no conflict of interest.

\section{References}

1. C. Ferraz, M. Eszlinger, R. Paschke, Current state and future perspective of molecular diagnosis of fine-needle aspiration biopsy of thyroid nodules. J. Clin. Endocrinol. Metab. 96(7), 2016-2026 (2011)

2. D.N. Poller, Z.W. Baloch, G. Fadda, S.J. Johnson, M. Bongiovanni, A. Pontecorvi, B. Cochand-Priollet, Thyroid FNA: new classifications and new interpretations. Cancer Cytopathol. 124(7), 457-466 (2016)

3. C. Durante, G. Costante, G. Lucisano, R. Bruno, D. Meringolo, A. Paciaroni, E. Puxeddu, M. Torlontano, S. Tumino, M. Attard, L. Lamartina, A. Nicolucci, S. Filetti, The natural history of benign thyroid nodules. JAMA 313(9), 926-935 (2015)

4. B.R. Haugen, E.K. Alexander, K.C. Bible, G.M. Doherty, S.J. Mandel, Y.E. Nikiforov, F. Pacini, G.W. Randolph, A.M. Sawka, M. Schlumberger, K.G. Schuff, S.I. Sherman, J.A. Sosa, D.L. Steward, R.M. Tuttle, L. Wartofsky, American Thyroid Association Management Guidelines for adult patients with thyroid nodules and differentiated thyroid cancer: The American Thyroid Association Guidelines Task Force on thyroid nodules and differentiated thyroid cancer. Thyroid 26(1), 1-133 (2016)

5. G. Grani, L. Lamartina, V. Ascoli, D. Bosco, F. Nardi, F. D'Ambrosio, A. Rubini, L. Giacomelli, M. Biffoni, S. Filetti, C. Durante, V. Cantisani, Ultrasonography scoring systems can rule out malignancy in cytologically indeterminate thyroid nodules. Endocrine 57(2), 256-261 (2017)

6. F. Nardi, F. Basolo, A. Crescenzi, G. Fadda, A. Frasoldati, F. Orlandi, L. Palombini, E. Papini, M. Zini, A. Pontecorvi, P. Vitti, Italian consensus for the classification and reporting of thyroid cytology. J. Endocrinol. Invest. 37(6), 593-599 (2014)

7. M. Nishino, Molecular cytopathology for thyroid nodules: a review of methodology and test performance. Cancer Cytopathol. 124(1), 14-27 (2016)

8. Y.E. Nikiforov, S.E. Carty, S.I. Chiosea, C. Coyne, U. Duvvuri, R.L. Ferris, W.E. Gooding, S.P. Hodak, S.O. LeBeau, N.P. Ohori, R.R. Seethala, M.E. Tublin, L. Yip, M.N. Nikiforova, Highly accurate diagnosis of cancer in thyroid nodules with follicular neoplasm/suspicious for a follicular neoplasm cytology by ThyroSeq v2 next-generation sequencing assay. Cancer 120(23), 3627-3634 (2014) 
9. Cancer Genome Atlas Research Network, Integrated genomic characterization of papillary thyroid carcinoma. Cell 159(3), 676-690 (2014)

10. P.S. Harper, Myotonic Dystrophy (Oxford University Press, Oxford, 2002)

11. M. Santoro, M. Masciullo, R. Pietrobono, G. Conte, A. Modoni, M.L. Bianchi, V. Rizzo, M.G. Pomponi, G. Tasca, G. Neri, G. Silvestri, Molecular, clinical, and muscle studies in myotonic dystrophy type 1 (DM1) associated with novel variant CCG expansions. J. Neurol. 260(5), 1245-1257 (2013)

12. I. Landa, T. Ibrahimpasic, L. Boucai, R. Sinha, J.A. Knauf, R.H. Shah, S. Dogan, J.C. Ricarte-Filho, G.P. Krishnamoorthy, B. Xu, N. Schultz, M.F. Berger, C. Sander, B.S. Taylor, R. Ghossein, I. Ganly, J.A. Fagin, Genomic and transcriptomic hallmarks of poorly differentiated and anaplastic thyroid cancers. J. Clin. Invest. 126(3), 1052-1066 (2016)

13. A. Verrienti, A. Carbone, P. Bellitti, M.C. Fabiano, R.F. De Rose, M. Maranghi, P. Lucia, C. Durante, F. Rosignolo, V. Pecce, M. Sponziello, C. Puppin, G. Costante, R. Bruno, A novel double mutation Val648ile and Val804leu of Ret proto-oncogene in multiple endocrine neoplasia type 2. Endocr. Pract. 21, 1248-1254 (2015)

14. A. Karunamurthy, F. Panebianco, S. J Hsiao, J. Vorhauer, M.N. Nikiforova, S. Chiosea, Y.E. Nikiforov, Prevalence and phenotypic correlations of EIF1AX mutations in thyroid nodules. Endocr. Relat. Cancer 23(4), 295-301 (2016)

15. M.C. Topf, Z.X. Wang, K. Furlong, J.L. Miller, M. Tuluc, E.A. Pribitkin, EIF1AX mutation in a patient with Hürthle cell carcinoma. Endocr. Pathol. 29(1), 27-29 (2016). https://doi.org/ 10.1007/s12022-017-9501-8

16. D.S. Olsen, E.M. Savner, A. Mathew, F. Zhang, T. Krishnamoorthy, L. Phan, A.G. Hinnebusch, Domains of eIF1A that mediate binding to eIF2, eIF3 and eIF5B and promote ternary complex recruitment in vivo. EMBO J. 22(2), 193-204 (2003)

17. P.D. Stenson, M. Mort, E.V. Ball, K. Evansm, M. Hayden, S. Heywoodm, M. Hussain, A.D. Phillips, D.N. Cooper, The Human Gene Mutation Database: towards a comprehensive repository of inherited mutation data for medical research, genetic diagnosis and next-generation sequencing studies. Hum. Genet. 136(6), 665-677 (2017).

18. A.K. Win, P.G. Perattur, J.S. Pulido, C.M. Pulido, N.M. Lindor, Increased cancer risks in myotonic dystrophy. Mayo Clin. Proc. 87(2), 130-135 (2012)

19. M.L. Bianchi, E. Leoncini, M. Masciullo, A. Modoni, S.M. Gadalla, R. Massa, E. Rastelli, C. Terracciano, G. Antonini, E. Bucci, A. Petrucci, S. Costanzi, M. Santoro, S. Boccia, G. Silvestri, Increased risk of tumor in DM1 is not related to exposure to common lifestyle risk factors. J. Neurol. 263(3), 492-498 (2016)

20. R. Fernández-Torrón, M. García-Puga, J.I. Emparanza, M. Maneiro, A.M. Cobo, J.J. Poza, J.B. Espinal, M. Zulaica, I. Ruiz, L. Martorell, D. Otaegui, A. Matheu, A. López de Munain, Cancer risk in DM1 is sex-related and linked to miRNA-200/141 downregulation. Neurology 87(12), 1250-1257 (2016) 